\title{
In vitro activity of dalbavancin and five comparator agents against common and uncommon Gram-positive organisms isolated from cancer patients
}

\author{
Kenneth VI Rolston ${ }^{1,2}$, Weiqun Wang ${ }^{2}$, Lior Nesher ${ }^{1}$, Samuel A Shelburne ${ }^{1}$ and Randall A Prince ${ }^{1,2}$ \\ Dalbavancin is a long acting, bactericidal lipoglycopeptide. Its in vitro activity was compared with that of vancomycin, \\ daptomycin, linezolid, trimethoprim/sulfamethoxazole (TMP/SMX) and levofloxacin against $241 \mathrm{Gram}$-positive organisms isolated \\ from cancer patients. The rank order of potency for the glycopeptides based on $\mathrm{MIC}_{90}\left(\mu \mathrm{g} \mathrm{ml}{ }^{-1}\right)$, that is, the concentration of \\ antimicrobial agent required to inhibit $90 \%$ of isolates tested was dalbavancin $\left(0.12 \mu \mathrm{g} \mathrm{ml}^{-1}\right)>$ daptomycin $\left(1.0 \mu \mathrm{g} \mathrm{ml}^{-1}\right)$ \\ $>$ vancomycin $\left(2.0 \mu \mathrm{g} \mathrm{ml}^{-1}\right)$ for coagulase-negative staphylococci and Staphylococcus aureus isolates (including methicillin- \\ susceptible (MSSA) and methicillin-resistant (MRSA) strains). Dalbavancin had potent activity against staphylococcal isolates \\ with vancomycin MICs $\geqslant 1.0 \mu \mathrm{g} \mathrm{ml}^{-1}$. TMP/SMX also had potent activity against staphylococci including methicillin-resistant \\ strains, whereas levofloxacin had moderate to poor anti-staphylococcal activity. Dalbavancin also exhibited more potent activity \\ than vancomycin and daptomycin against Bacillus spp., Corynebacterium spp., Micrococcus spp. and various streptococci \\ (including Streptococcus pneumoniae, viridans group streptococci (VGS), beta-hemolytic streptococci and gamma-hemolytic \\ streptococci). MBC determinations showed that dalbavancin had potent bactericidal activity against MRSA with no tolerance \\ being detected. These data suggest that dalbavancin may be considered as an alternative to vancomycin, especially in \\ institutions wherein a substantial proportion of infections are caused by organisms with vancomycin MICs $\geqslant 1.0 \mu \mathrm{g} \mathrm{ml}^{-1}$. \\ The Journal of Antibiotics (2016) 69, 381-387; doi:10.1038/ja.2015.120; published online 2 December 2015
}

\section{INTRODUCTION}

Infections remain the most common medical complications seen in patients with cancer. Although the frequency and severity of infection is greater in patients with neutropenia (absolute neutrophil count or ANC $<500 \mathrm{~mm}^{-3}$ ), the vast majority of cancer patients who develop infections are not neutropenic. Gram-positive organisms are the predominant bacterial pathogens in this setting, globally. ${ }^{1-4}$ Staphylococcus species (coagulase-negative staphylococci (CoNS), Staphylococcus aureus (S. aureus), both methicillin-susceptible and methicillin-resistant strains) are isolated most often, but a wide spectrum of Gram-positive bacteria such as Bacillus species, Corynebacterium species, Micrococcus species, viridans group streptococci (VGS) and beta-hemolytic streptococci are also encountered. ${ }^{5}$ Monomicrobial bloodstream infections (including catheter-related infections) are probably the most frequent Gram-positive infections. Other common sites of infection include the skin and skin structures or SSIs (including surgical site infections), bones and joints, the respiratory tract and the intestinal tract. In addition, a substantial proportion of polymicrobial infections have a Gram-positive component. ${ }^{6}$

For many decades vancomycin has been the agent of choice for the treatment of documented Gram-positive infections in most cancer-treatment centers. It is also recommended for empiric usage when Gram-positive infections are strongly suspected. ${ }^{7,8}$ These recommendations may now be outdated at some institutions. Many centers have documented declining vancomycin in vitro activity (the so-called MIC creep), vancomycin tolerance $(\mathrm{MBC} \geqslant 32$ times the MIC), hetero-resistance, overt vancomycin resistance and lack of bactericidal activity especially among methicillin-resistant $S$. aureus (MRSA) isolates. ${ }^{9-12}$ Delayed responses and overt clinical failures have been reported in staphylococcal infections caused by isolates with vancomycin MICs of $1.0-2.0 \mu \mathrm{g} \mathrm{ml}^{-1}$, which are still regarded as vancomycin susceptible. ${ }^{9,13}$ Reports such as these led to the lowering of vancomycin susceptibility/resistance breakpoints for S. aureus by the Clinical Laboratory Standards Institute (CLSI). ${ }^{14}$ A recently published meta-analysis documented that infections caused by $S$. aureus isolates with vancomycin MICs of $>1.5 \mu \mathrm{g} \mathrm{ml}^{-1}$ were associated with increased mortality irrespective of the source of infection or the MIC-testing method. ${ }^{15}$ In addition, clinical data from our center (an NCI-designated Comprehensive Cancer Center) has shown a high vancomycin treatment failure rate in cancer patients with MRSA bloodstream infections. ${ }^{16} \mathrm{~A}$ vancomycin MIC of $\geqslant 2.0 \mu \mathrm{g} \mathrm{ml}^{-1}$ was an independent predictor of MRSA mortality in this study. Recent

\footnotetext{
${ }^{1}$ Department of Infectious Diseases, Infection Control, and Employee Health, The University of Texas, MD Anderson Cancer Center, Houston, TX, USA and ${ }^{2}$ The University of Houston College of Pharmacy, Houston, TX, USA

Correspondence: Professor KVI Rolston, The University of Texas, MD Anderson Cancer Center, Department of Infectious Diseases, Infection Control and Employee Health, 1515 Holcombe Boulevard (Unit 1460), Houston, TX 77030, USA.

E-mail: krolston@mdanderson.org

Received 24 August 2015; revised 16 October 2015; accepted 1 November 2015; published online 2 December 2015
} 
guidelines for the treatment of MRSA infections recommend that clinical response should determine the use of vancomycin for isolates with an MIC of $\leqslant 2.0 \mu \mathrm{g} \mathrm{ml}^{-1}$, whereas an alternative to vancomycin should be used for infections caused by isolates with an MIC $>2.0 \mu \mathrm{g} \mathrm{ml}^{-1} .17$

Agents such as linezolid, daptomycin and telavancin are considered alternatives to vancomycin, but clinical data for these agents in cancer patients are scant, and each of these agents is associated with some limitations such as toxicity, and lack of bactericidal activity. ${ }^{18-20}$ Dalbavancin is a novel semisynthetic lipoglycopeptide with considerable potency against a wide spectrum of Gram-positive pathogens, many of which cause infections in cancer patients. ${ }^{21-23}$ It possesses a long half-life that enables weekly administration. We believe that dalbavancin has a potential role in the treatment of some Gram-positive infections in cancer patients. Consequently, we compared its in vitro activity to vancomycin, daptomycin, linezolid, trimethoprim/sulfamethoxazole (TMP/SMX) and levofloxacin, against recent clinical isolates obtained from cancer patients treated at our institution.

\section{RESULTS}

The comparative in vitro activities of dalbavancin, vancomycin, daptomycin, linezolid, TMP/SMX and levofloxacin against 241 isolates representing at least 15 bacterial species are presented in Table 1.

\section{Staphylococci}

Of the 50 CoNS isolates tested, seven, all Staphylococcus lugdunensis, were identified to species level. All seven were inhibited by 0.03 $\mu \mathrm{g} \mathrm{ml}^{-1}$ of dalbavancin, which had lower MICs than all the comparator agents including vancomycin. Nevertheless, only one isolate had a vancomycin MIC of $1.0 \mu \mathrm{g} \mathrm{ml}^{-1}$. These isolates were also susceptible to all comparator agents. Of the remaining 43 CoNS isolates, 25 (58\%) were methicillin resistant (MR-CoNS). Dalbavancin had a bimodal pattern of activity against MR-CoNS inhibiting 19 of 25 isolates $(76 \%)$ at $\leqslant 0.06 \mu \mathrm{g} \mathrm{ml}^{-1}$, and 6 isolates $(20 \%)$ at $0.12 \mu \mathrm{g} \mathrm{ml}^{-1}$. One isolate had a dalbavancin MIC of $0.25 \mu \mathrm{g} \mathrm{ml}{ }^{-1}$. In contrast, only two isolates $(8 \%)$ had vancomycin MICs $<1.0 \mu \mathrm{g} \mathrm{ml}^{-1}$ although no overt vancomycin resistance was detected. All isolates were susceptible to daptomycin and TMP/SMX, one isolate was resistant to linezolid (MIC $128.0 \mu \mathrm{g} \mathrm{ml}^{-1}$ ), whereas levofloxacin had poor activity against MR-CoNS. Similarly, 15 of 18 (83\%) methicillin-susceptible isolates (MS-CoNS) were inhibited by $\leqslant 0.06 \mu \mathrm{g} \mathrm{ml}^{-1}$ of dalbavancin and 3 (17\%) had an MIC of $0.12 \mu \mathrm{g} \mathrm{ml}^{-1}$. In contrast, 10 of $18 \mathrm{MS}-\mathrm{CoNS}$ isolates $(56 \%)$ had vancomycin MICs $\geqslant 1.0 \mu \mathrm{g} \mathrm{ml}^{-1}$. All 18 isolates were susceptible to daptomycin, TMP/SMX and linezolid, while levofloxacin had moderate activity against MS-CoNS. Although all CoNS isolates were susceptible to daptomycin, only $6(12 \%)$ had an MIC $<0.5 \mu \mathrm{g} \mathrm{ml}^{-1}$.

Of the $85 \mathrm{~S}$. aureus isolates tested $35(41 \%)$ were methicillin susceptible (MSSA). Dalbavancin again exhibited potent but bimodal activity against these isolates inhibiting $31(86 \%)$ at $\leqslant 0.03 \mu \mathrm{g} \mathrm{ml}^{-1}$, 3 at $0.12 \mu \mathrm{g} \mathrm{ml}^{-1}$, whereas one isolate was non-susceptible with an MIC of $0.25 \mu \mathrm{g} \mathrm{ml}^{-1}$. A substantial proportion of these isolates (20, or $57 \%$ ) had a vancomycin MIC $\geqslant 1.0 \mu \mathrm{g} \mathrm{ml}^{-1}$, although all isolates were vancomycin susceptible. All isolates were also susceptible to daptomycin, TMP/SMX and linezolid, and 32 (91\%) were inhibited by $\leqslant 0.5 \mu \mathrm{g} \mathrm{ml}^{-1}$ of levofloxacin. Dalbavancin had potent activity against most MRSA isolates inhibiting $44(88 \%)$ at $\leqslant 0.06 \mu \mathrm{g} \mathrm{ml}^{-1}$, whereas four isolates had an MIC of $0.12 \mu \mathrm{g} \mathrm{ml}^{-1}$ and one isolate had an MIC of $0.25 \mu \mathrm{g} \mathrm{ml}^{-1}$ (Table 2). In contrast 35 isolates (70\%) had a vancomycin $\mathrm{MIC} \geqslant 1.0 \mu \mathrm{g} \mathrm{ml}^{-1}$, although all isolates were susceptible to it. All isolates were also susceptible to daptomycin (range $0.25-1.0 \mu \mathrm{g} \mathrm{ml}^{-1}$ ), linezolid (range $0.5-2.0 \mu \mathrm{g} \mathrm{ml}^{-1}$ ) and TMP/SMX. Levofloxacin had poor activity against MRSA.

\section{Streptococci}

Dalbavancin exhibited potent activity against VGS inhibiting all 20 isolates at $\leqslant 0.06 \mu \mathrm{g} \mathrm{ml}^{-1}$ (range $0.008-0.06 \mu \mathrm{g} \mathrm{ml}^{-1}$ ). All VGS isolates were susceptible to vancomycin, daptomycin and TMP/SMX, but their MICs were several fold higher than dalbavancin. Levofloxacin had poor activity against VGS. Of the seven Streptococcus pneumoniae (S. pneumoniae) isolates tested, all were inhibited by $\leqslant 0.016 \mu \mathrm{g} \mathrm{ml}^{-1}$ of dalbavancin, $\leqslant 0.25 \mu \mathrm{g} \mathrm{ml}^{-1}$ of vancomycin, $\leqslant 0.5 \mu \mathrm{g} \mathrm{ml}^{-1}$ of daptomycin and $\leqslant 1.0 \mu \mathrm{g} \mathrm{ml}^{-1}$ of linezolid. All isolates were also susceptible to TMP/SMX and levofloxacin. Eleven beta-hemolytic streptococci (three Group A and eight Group B) and 10 gamma hemolytic streptococci were available for testing. Dalbavancin had potent in vitro activity against all these isolates, as did all comparator agents except levofloxacin that had poor activity against gamma hemolytic strains.

\section{Other Gram-positive organisms}

Dalbavancin had potent activity against Bacillus spp. inhibiting 19 of 21 isolates $(90 \%)$ at $\leqslant 0.06 \mu \mathrm{g} \mathrm{ml}^{-1}$ while one isolate had an MIC of $0.25 \mu \mathrm{g} \mathrm{ml}^{-1}$. Although all 21 were susceptible to vancomycin, $9(43 \%)$ had MICs $\geqslant 1.0 \mu \mathrm{g} \mathrm{ml}^{-1}$. All these isolates were susceptible to the four other comparator agents. All 23 Corynebacterium spp. isolates were inhibited by $\leqslant 0.03 \mu \mathrm{g} \mathrm{ml}^{-1}$ of dalbavancin (range $0.008-0.03 \mu \mathrm{g} \mathrm{ml}^{-1}$ ). Although all these isolates were susceptible to vancomycin, daptomycin, TMP/SMX and linezolid, the MICs for these three agents were several fold higher than dalbavancin. Levofloxacin had moderate activity against Corynebacterium spp. Similarly, dalbavancin was more potent against Micrococcus spp. than the comparator agents including vancomycin and daptomycin. Dalbavancin inhibited the two Rhodococcus equi isolates and the lone Listeria monocytogenes isolate at $0.03 \mu \mathrm{g} \mathrm{ml}^{-1}$.

We determined the proportion of all Gram-positive isolates tested that had vancomycin MIC $\geqslant 1.0 \mu \mathrm{g} \mathrm{ml}^{-1}$ (Table 3). The highest proportion of organisms exhibiting such MICs was seen among MR-CoNS (92\%), followed by MRSA (70\%), MSSA (57\%), MS-CoNS (56\%) and Bacillus spp. (43\%). All other species tested were inhibited by $\leqslant 0.5 \mu \mathrm{g} \mathrm{ml}^{-1}$ of vancomycin. As the clinical significance of such isolates has only been established for MRSA, we compared and contrasted the cumulative activities of dalbavancin and comparator agents only against MRSA isolates (Table 2). All of the 35 isolates with vancomycin MICs $\geqslant 1.0 \mu \mathrm{g} \mathrm{ml}^{-1}$ were inhibited by dalbavancin at concentrations of $\leqslant 0.12 \mu \mathrm{g} \mathrm{ml}^{-1}$. In addition, MBCs were determined for dalbavancin, vancomycin and daptomycin against 50 MRSA isolates. These results are shown in Table 4. All three agents exhibited good bactericidal activity against these isolates. The MBCs were $\leqslant 4$-fold the MICs for 42 isolates $(84 \%)$ for dalbavancin, 40 isolates (80\%) for vancomycin and 48 isolates (96\%) for daptomycin, respectively. No tolerance ( $\mathrm{MBC} \geqslant 32$ times the MIC) to dalbavancin or daptomycin was detected, and only one isolate $(2 \%)$ was tolerant to vancomycin.

\section{DISCUSSION}

Gram-positive organisms remain the predominant bacterial pathogens in neutropenic and non-neutropenic cancer patients at most cancer-treatment centers, although some institutions are reporting a resurgence of Gram-negative infections especially in patients not receiving fluoroquinolone prophylaxis. ${ }^{1-3,24}$ Currently, $\sim 70-80 \%$ of 
Table 1 In vitro activity of dalbavancin and five comparator agents against Gram-positive isolates from cancer patients

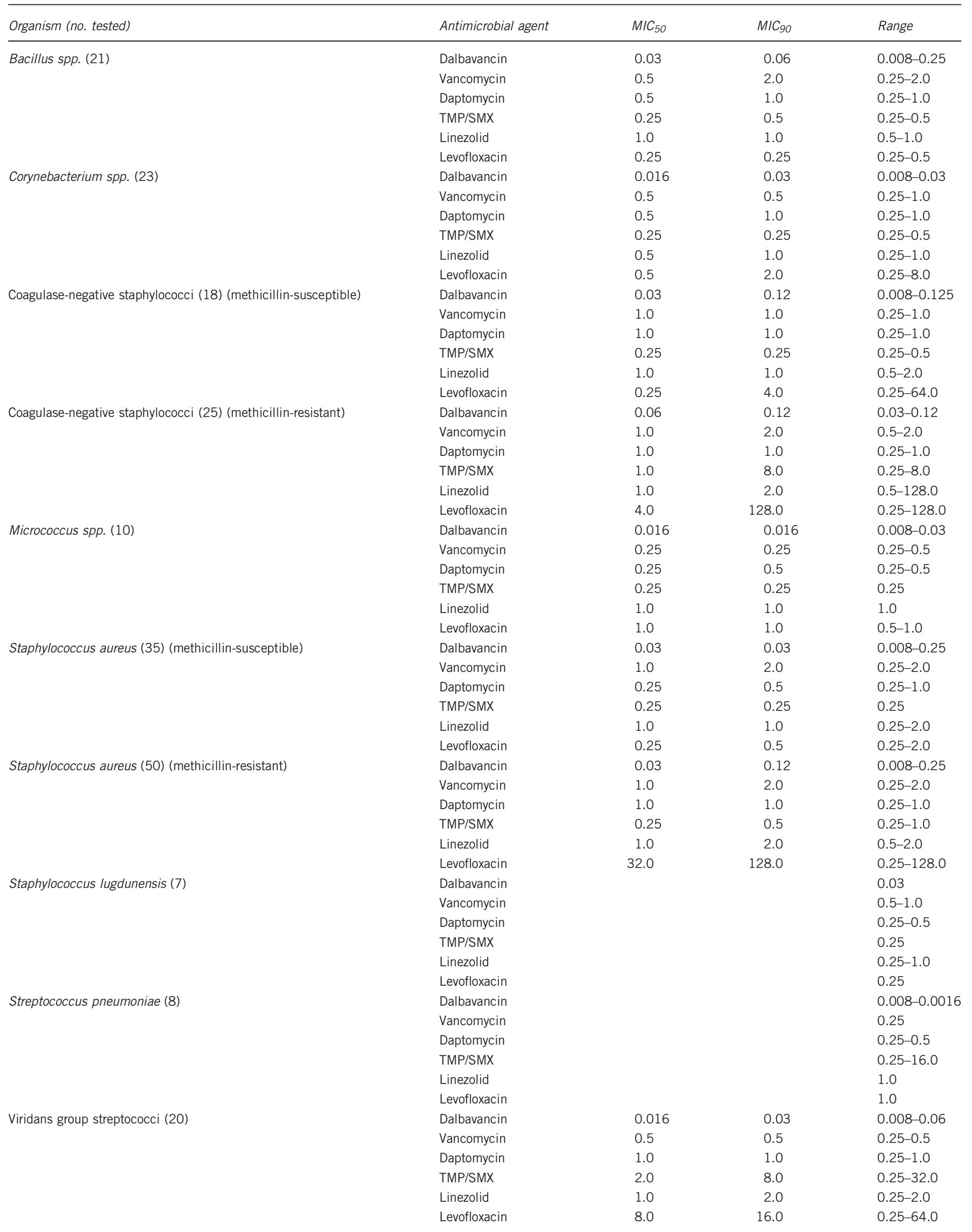


Table 1 (Continued)

\begin{tabular}{|c|c|c|c|c|}
\hline Beta-hemolytic streptococci (11 ) & Dalbavancin & & & $0.016-0.06$ \\
\hline \multirow[t]{4}{*}{ Group A (3) } & Vancomycin & & & $0.25-0.5$ \\
\hline & TMP/SMX & & & $0.25-0.5$ \\
\hline & Linezolid & & & 1.0 \\
\hline & Levofloxacin & & & $0.25-0.5$ \\
\hline \multirow{4}{*}{ Group B (8) } & Daptomycin & & & $0.25-1.0$ \\
\hline & SMP/SMX & & & $0.25-1.0$ \\
\hline & Linezolid & & & $0.25-1.0$ \\
\hline & Levofloxacin & & & $0.25-1.0$ \\
\hline \multirow{2}{*}{$\begin{array}{l}\text { Gamma hemolytic } \\
\text { streptococci (10) }\end{array}$} & Dalbavancin & 0.016 & 0.06 & $0.016-0.06$ \\
\hline & Vancomycin & 0.5 & 0.5 & $0.25-0.5$ \\
\hline Listeria & Dalbavancin & & & 0.03 \\
\hline \multirow[t]{5}{*}{ monocytogenes (2) } & Vancomycin & & & 0.5 \\
\hline & Daptomycin & & & $0.25-0.5$ \\
\hline & SMP/SMX & & & 0.25 \\
\hline & Linezolid & & & 0.5 \\
\hline & Levofloxacin & & & 0.25 \\
\hline \multirow[t]{5}{*}{ Rhodococcus equi ( 1 ) } & Dalbavancin & & & 0.03 \\
\hline & Vancomycin & & & 0.25 \\
\hline & Daptomycin & & & 32.0 \\
\hline & SMP/SMX & & & 0.5 \\
\hline & Linezolid & & & 2.0 \\
\hline
\end{tabular}

Abbreviation: SMP/SMX, trimethoprim/sulfamethoxazole.

bacteremias (including central line-related bloodstream infections) and $\sim 35-50 \%$ of infections at all other sites have a Gram-positive component. CoNS are isolated most often followed by S. aureus, VGS and Enterococcus species. Other Gram-positive organisms (Bacillus spp., Corynebacterium spp., Micrococcus spp. and beta-hemolytic streptococci) are isolated with varying frequency, whereas Rhodococcus equi and Listeria monocytogenes are quite infrequent. Consequently, when empiric Gram-positive coverage is considered necessary in cancer patients, the agent used needs to have activity not just against the staphylococci and VGS but against a much wider spectrum of Gram-positive pathogens. In addition, many of the organisms listed above are resistant to the fluoroquinolones and beta-lactams, agents that are often used for infection prevention and/or treatment of infections in cancer patients.

Until quite recently, vancomycin was considered to be the agent of choice for the treatment of Gram-positive infections in patients with cancer. In fact, previous versions of the Infectious Diseases Society of America (IDSA) guidelines for the management of febrile neutropenic patients recommend the use of vancomycin when such infections are documented or strongly suspected. ${ }^{24,25}$ However, over the past 8-10 years, several reports have been published documenting vancomycin failures in staphylococcal infections caused by strains considered to be susceptible to it, but with MICs $\geqslant 1.0 \mu \mathrm{g} \mathrm{ml}^{-1.9,13}$ Institutions worldwide, including our own, have documented an upward MIC creep with a substantial proportion of $S$. aureus and other organisms being vancomycin susceptible based on current CLSI break points, but with MICs of $1.0 \mu \mathrm{g} \mathrm{ml}^{-1}$ or more. Diminished vancomycin bactericidal activity has been documented against such isolates, probably becuase of altered quorum sensing and other mechanisms. ${ }^{13}$ These reports have raised concerns that vancomycin may no longer be the agent of choice for the treatment of infections caused by such isolates, especially in neutropenic patients in whom bactericidal agents are generally preferred. ${ }^{26}$ These concerns led us to review treatment and outcomes of cancer patients with MRSA infections at our institution between 2001 and 2009. ${ }^{16}$ We found a high treatment failure rate (52\%) for vancomycin and an independent predictor of infectionrelated mortality was an infection caused by MRSA strains with MICs of $>2.0 \mu \mathrm{g} \mathrm{ml}^{-1}$. In addition, a recent review of SSIs in our patients with solid organ malignancies demonstrated that MRSA was the predominant organism isolated and that $65 \%$ of MRSA isolates had a vancomycin MIC $\geqslant 1.0 \mu \mathrm{g} \mathrm{ml}^{-1} \cdot{ }^{27} \mathrm{On}$ the basis of this experience we have developed institutional clinical practice guidelines that recommend the use of alternative agents (for example, daptomycin or linezolid) when such infections are encountered in our patients.

Both linezolid and daptomycin are associated with limitations, leaving room for the development and evaluation of other agents. Linezolid is bacteriostatic, and is associated with myelotoxicity, making it less than ideal especially in patients with underlying hematological malignancies and in hematopoietic stem cell transplantation recipients. ${ }^{28}$ There have also been reports of increasing resistance to 
Table 2 Cumulative proportions of 50 MRSA isolates inhibited by increasing concentrations of antimicrobial agents

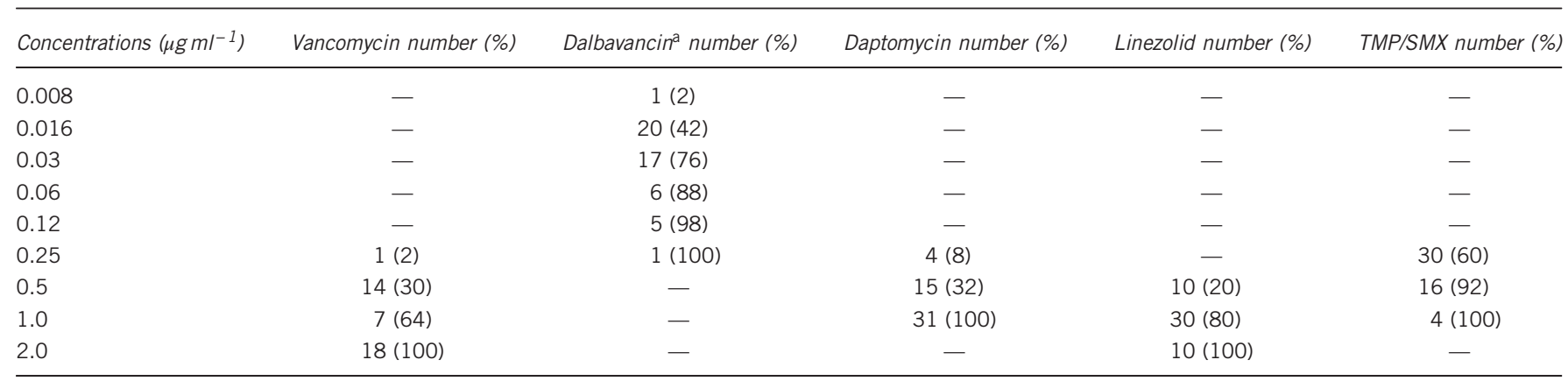

aDalbavancin inhibited all 35 isolates with vancomycin MICs $\geqslant 1.0 \mu \mathrm{g} \mathrm{ml}-1$ at concentrations $\leqslant 0.12 \mu \mathrm{g} \mathrm{ml}-1$.

Table 3 Proportion Gram-positive of isolates with vancomycin MICs $\geqslant 1.0 \mu \mathrm{g} \mathrm{ml}^{-1}$

\begin{tabular}{lccc}
\hline Bacterial species $^{a}$ & No. tested & Vancomycin MIC $\geqslant 1.0 \mu \mathrm{g} \mathrm{ml}^{-1}$ no. & $\%$ \\
\hline Bacillus species & $(21)$ & 9 & $(43)$ \\
MS-CoNS & $(18)$ & 10 & $(56)$ \\
MR-CoNS & $(25)$ & 23 & $(92)$ \\
S. lugdunensis & $(7)$ & 1 & $(14)$ \\
MSSA & $(35)$ & 20 & $(57)$ \\
MRSA & $(50)$ & 35 & $(70)$ \\
Corynebacterium species & $(23)$ & 2 & $(9)$ \\
\hline
\end{tabular}

Abbreviations: MR-CoNS, methicillin-resistant coagulase-negative staphylococci; MS-CoNS, methicillin-susceptible coagulase-negative staphylococci. MRSA, methicillin-resistant $S$, aureus; MSSA, methicillin susceptible S. aureus.

aAll other species tested were inhibited by $\leqslant 0.5 \mu \mathrm{g} \mathrm{ml}^{-1}$ of vancomycin.

linezolid among staphylococcal isolates, often associated with prolonged usage..$^{29}$ Although daptomycin does not have these limitations, it is inactivated by pulmonary surfactant. A substantial proportion of neutropenic patients (25-40\%) develops pulmonary infiltrates precluding the use of daptomycin in this setting. TMP/SMX is not used for empiric therapy in neutropenic patients, but is sometimes used for the treatment of documented Gram-positive infections including those caused by MRSA. It can be associated with substantial toxicity and there is a general reluctance to use this agent particularly in neutropenic patients and hematopoietic stem cell transplantation recipients. Levofloxacin is currently the most common agent used for antimicrobial prophylaxis, and as confirmed by our in vitro data, it has poor activity against Gram-positive organisms.

Unlike other studies evaluating the in vitro activity of dalbavancin, our study tested isolates obtained exclusively from patients with cancer, many of whom were neutropenic, and all of whom had prior exposure to antimicrobial agents. Our data demonstrate that at our institution, the majority of staphylococcal isolates and a substantial proportion of other Gram-positive isolates have vancomycin MICs of $\geqslant 1.0 \mu \mathrm{g} \mathrm{ml}^{-1}$. Our data also demonstrate that dalbavancin has potent in vitro activity against the majority of Gram-positive organisms that cause infections in cancer patients, including those with vancomycin MICs of $\geqslant 1.0 \mu \mathrm{g} \mathrm{ml}^{-1}$, and that this activity is several fold greater than comparator agents including daptomycin, linezolid, TMP/SMX and levofloxacin against most species tested. This could have clinical significance as the response rate to vancomycin for MRSA infections caused by strains with MICs $\geqslant 1.0 \mu \mathrm{g} \mathrm{ml}^{-1}$ are suboptimal. Only one MRSA isolate had a dalbavancin MIC of $0.25 \mu \mathrm{g} \mathrm{ml}^{-1}$ (considered non-susceptible). This is in line with a recently published global
Table 4 MBC:MIC ratios for vancomycin, dalbavancin, and daptomycin against 50 MRSA isolates

\begin{tabular}{lccc}
\hline $\begin{array}{l}\text { MBC:MIC } \\
\text { ratio }\end{array}$ & $\begin{array}{c}\text { Vancomycin number } \\
(\%)\end{array}$ & $\begin{array}{c}\text { Dalbavancin number } \\
\text { (\%) }\end{array}$ & $\begin{array}{c}\text { Daptomycin number } \\
\text { (\%) }\end{array}$ \\
\hline $1: 1$ & $6(12)$ & $10(20)$ & $19(38)$ \\
$2: 1$ & $28(56)$ & $24(48)$ & $23(46)$ \\
$4: 1$ & $6(12)$ & $8(16)$ & $6(12)$ \\
$8: 1$ & $4(8)$ & $8(16)$ & $2(4)$ \\
$16: 1$ & $4(8)$ & - & - \\
$\geqslant 32: 1$ & $1(2)$ & - & - \\
\hline
\end{tabular}

surveillance study, in which 62195 non-duplicate S. aureus isolates were tested. Dalbavancin demonstrated sustained potency against the vast majority of these isolates, including isolates that were nonsusceptible to daptomycin, linezolid and tigecycline. ${ }^{30}$ Only $0.35 \%$ of these isolates had a dalbavancin MIC of either 0.25 or 0.5 .

Dalbavancin has been evaluated in two identical, international, multicenter, randomized trials for the treatment of acute bacterial skin and skin-stricture infections (ABSSSI), using a single 1000-mg dose followed by a 500-mg dose 1 week later, and was not inferior to parenteral vancomycin followed by oral linezolid. ${ }^{31}$ It was subsequently approved by the US FDA for this indication in adults. ${ }^{32}$ It has also been evaluated for the treatment of catheter-related bloodstream infections using a similar dosage schedule, with an overall success rate of $87 \% .{ }^{33}$ A recently completed phase 3 double-blind, double-dummy trial comparing a single 1500-mg dose to the above-mentioned two-dose regimen for the treatment of ABSSSI in 698 patients has demonstrated that the 1500 -mg single-dose regimen is non-inferior to the standard two-dose regimen. ${ }^{34}$ Previously published pharamacokinetic data indicate that dalbavancin should maintain bactericidal activity using both dosage regimens. ${ }^{35,36}$

Dalbavancin has not been formally evaluated in neutropenic or other immunosuppressed cancer patients, as the studies mentioned above excluded such patients. Our in vitro data demonstrate that dalbavancin has potent activity against most Gram-positive pathogens isolated from cancer patients. These include organisms that commonly cause complicated skin and skin-structure infections including SSIs, and those with reduced susceptibility to vancomycin. Dalbavancin could have an advantage over other agents because of its long half-life, which eliminates the need for frequent dosing and may facilitate outpatient management of patients with such infections. As mentioned previously, agents such as vancomycin are used often to provide 
empiric Gram-positive coverage in neutropenic patients. One of the caveats of such usage is discontinuation of the agent after $24-48 \mathrm{~h}$ if no Gram-positive organisms have been isolated. Consequently, we caution against the empiric use of dalbavancin in this setting due to its long half-life and lack of long-term safety data. We believe that clinical trials to evaluate its efficacy in this unique patient population are warranted.

\section{METHODS}

\section{Organisms}

The organisms included in this study were recovered from clinical cultures submitted to our institutional microbiology laboratory from patients with various hematological malignancies and solid tumors, treated between 2013 and 2014, and were subsequently stored in our research microbiology laboratory's 'bug bank' for future use. These were not consecutive isolates but were chosen in an arbitrary manner in order to avoid any seasonal variation. To avoid duplication, only one isolate per patient per episode of infection was tested. The majority of the isolates ( $>90 \%$ ) were from blood culture specimens. The rest were from other significant clinical sites including cerebrospinal, pleural, synovial fluids and wounds. These were generally, but not always, the first isolate of the clinical episode. Approval for this study by the institutional review board (IRB) was obtained before collection of organisms and susceptibility testing (MDACC protocol \# PA-12-0352).

\section{Antimicrobial agents}

Dalbavancin was obtained from the manufacturer (Durata Therapeutics, now Allergan plc, Branford, CT, USA) in the form of standard laboratory powder for in vitro testing. All five comparator agents were purchased from commercial vendors. The compounds were stored and prepared for use according to the labeled instructions for each product.

\section{Susceptibility testing}

Susceptibility testing was performed using the broth microdilution methodology in accordance with CLSI guidelines. ${ }^{37}$ Briefly, susceptibility testing was conducted using in-house prepared 96-well panels. Stock solutions were prepared using cation-adjusted Mueller-Hinton broth and Mueller-Hinton broth with $5 \%$ lysed horse blood as appropriate. Dalbavancin was tested with each media type supplemented with $0.002 \%$ polysorbate- 80 . For daptomycin, further supplementation with calcium was done as recommended by CLSI. MICs were interpreted using CLSI M100-S24 breakpoints, except for dalbavancin. Where applicable, the US FDA approved MIC breakpoints for dalbavancin were used. Appropriate ATCC control strains (S. aureus ATCC 29213, and S. pneumoniae ATCC 49619) were used during each susceptibility test run in order to ensure the accuracy and validity of our results. Drug concentrations that inhibited 50 and $90 \%$ of isolates $\left(\mathrm{MIC}_{50}\right.$ and $\mathrm{MIC}_{90}$ values, respectively) were calculated only if 10 or more isolates of an individual species were available for testing. When fewer than 10 isolates were available, MIC ranges were reported. MBC determinations were done for MRSA isolates using the plating and colony-count procedures as outlined in the CLSI M26-A document.

\section{CONFLICT OF INTEREST}

The authors declare no conflict of interest.

\section{ACKNOWLEDGEMENTS}

This study was supported by a grant from Durata Therapeutics (now Allergan plc, Branford, CT, USA).

1 Wisplinghoff, H., Seifert, H., Wenzel, R. P. \& Edmond, M. B. Current trends in the epidemiology of nosocomial bloodstream infections in patients with hematological malignancies and solid neoplasms in hospitals in the United States. Clin. Infect. Dis. 36, 1103-1110 (2003).
2 Klastersky, J. et al. Bacteraemia in febrile neutropenic cancer patients. Int. J. Antimicrob. Agents 30(Suppl 1), S51-S59 (2007).

3 Safdar, A. \& Armstrong, D. Infections in patients with hematologic neoplasms and hematopoietic stem cell transplantation: neutropenia, humoral, and splenic defects. Clin. Infect. Dis. 53, 798-806 (2011).

4 Nesher, L. \& Rolston, K. V. The current spectrum of infection in cancer patients with chemotherapy related neutropenia. Infection 42, 5-13 (2014).

5 Rolston, K. V., Kapadia, M., Tarrand, J., Coyle, E. \& Prince, R. A. Spectrum of grampositive bacteraemia and in vitro activities of daptomycin, linezolid and vancomycin against organisms isolated from cancer patients. Int. J. Antimicrob. Agents 41, 516-520 (2013).

6 Rolston, K. V., Bodey, G. P. \& Safdar, A. Polymicrobial infection in patients with cancer: an underappreciated and underreported entity. Clin. Infect. Dis. 45, 228-233 (2007).

7 Moellering, R. C. Jr. Vancomycin: a 50-year reassessment. Clin. Infect. Dis. 42 (Suppl 1), S3-S4 (2006).

8 Freifeld, A. G. et al. Clinical practice guideline for the use of antimicrobial agents in neutropenic patients with cancer: 2010 update by the infectious diseases society of america. Clin. Infect. Dis. 52, e56-e93 (2011).

9 Sakoulas, G. et al. Relationship of MIC and bactericidal activity to efficacy of vancomycin for treatment of methicillin-resistant Staphylococcus aureus bacteremia. J. Clin. Microbiol. 42, 2398-2402 (2004).

10 Safdar, A. \& Rolston, K. V. Vancomycin tolerance, a potential mechanism for refractory gram-positive bacteremia observational study in patients with cancer. Cancer 106, 1815-1820 (2006).

11 Steinkraus, G., White, R. \& Friedrich, L. Vancomycin MIC creep in non-vancomycinintermediate Staphylococcus aureus (VISA), vancomycin-susceptible clinical methicillin-resistant S. aureus (MRSA) blood isolates from 2001-05. J. Antimicrob. Chemother. 60, 788-794 (2007).

12 Pillai, S. K. et al. Development of reduced vancomycin susceptibility in methicillinsusceptible Staphylococcus aureus. Clin. Infect. Dis. 49, 1169-1174 (2009).

13 Moise-Broder, P. A. et al. Accessory gene regulator group II polymorphism in methicillin-resistant Staphylococcus aureus is predictive of failure of vancomycin therapy. Clin. Infect. Dis. 38, 1700-1705 (2004).

14 Tenover, F. C. \& Moellering, R. C. Jr. The rationale for revising the Clinical and Laboratory Standards Institute vancomycin minimal inhibitory concentration interpretive criteria for Staphylococcus aureus. Clin. Infect. Dis. 44, 1208-1215 (2007).

15 van Hal, S. J., Lodise, T. P. \& Paterson, D. L. The clinical significance of vancomycin minimum inhibitory concentration in Staphylococcus aureus infections: a systematic review and meta-analysis. Clin. Infect. Dis. 54, 755-771 (2012).

16 Mahajan, S. N. et al. Characteristics and outcomes of methicillin-resistant staphylococcus aureus bloodstream infections in patients with cancer treated with vancomycin: 9-year experience at a comprehensive cancer center. Oncologist 17, 1329-1336 (2012).

$17 \mathrm{Liu}, \mathrm{C}$. et al. Clinical practice guidelines by the infectious diseases society of america for the treatment of methicillin-resistant Staphylococcus aureus infections in adults and children: executive summary. Clin. Infect. Dis. 52, 285-292 (2011).

18 Jaksic, B. et al. Efficacy and safety of linezolid compared with vancomycin in a randomized, double-blind study of febrile neutropenic patients with cancer. Clin. Infect. Dis. 42, 597-607 (2006).

19 Rolston, K. V. et al. Daptomycin use in neutropenic patients with documented grampositive infections. Support Care Cancer 22, 7-14 (2014).

20 Rolston, K. t. et al. In vitro activity of telavancin compared with vancomycin and linezolid against Gram-positive organisms isolated from cancer patients. J. Antibiot. (Tokyo) 67, 505-509 (2014).

21 Karlowsky, J. A. et al. In vitro activity of dalbavancin and telavancin against staphylococci and streptococci isolated from patients in Canadian hospitals: results of the CANWARD 2007-2009 study. Diagn. Microbiol. Infect. Dis. 69, 342-347 (2011)

22 Jones, R. N. \& Stilwell, M. G. Comprehensive update of dalbavancin activity when tested against uncommonly isolated streptococci, Corynebacterium spp., Listeria monocytogenes, and Micrococcus spp. (1357 strains). Diagn. Microbiol. Infect. Dis. 76, 239-240 (2013).

23 Jones, R. N., Sader, H. S. \& Flamm, R. K. Update of dalbavancin spectrum and potency in the USA: report from the SENTRY Antimicrobial Surveillance Program (2011). Diagn. Microbiol. Infect. Dis. 75, 304-307 (2013).

24 Montassier, E., Batard, E., Gastinne, T., Potel, G. \& de La Cochetière, M. F. Recent changes in bacteremia in patients with cancer: a systematic review of epidemiology and antibiotic resistance. Eur. J. Clin. Microbiol. Infect. Dis. 32, 841-850 (2013).

25 Hughes, W. T. et al. 1997 guidelines for the use of antimicrobial agents in neutropenic patients with unexplained fever. Infectious Diseases Society of America. Clin. Infect. Dis. 25, 551-573 (1997).

26 Finberg, R. W. et al. The importance of bactericidal drugs: future directions in infectious disease. Clin. Infect. Dis. 39, 1314-1320 (2004).

27 Rolston, K. V., Nesher, L. \& Tarrand, J. T. Current microbiology of surgical site infections in patients with cancer: a retrospective review. Infect. Dis. Ther. 3, 245-256 (2014).

28 Gerson, S. L. et al. Hematologic effects of linezolid: summary of clinical experience. Antimicrob. Agents Chemother. 46, 2723-2726 (2002).

$29 \mathrm{Gu}$, B., Kelesidis, T., Tsiodras, S., Hindler, J. \& Humphries, R. M. The emerging problem of linezolid-resistant Staphylococcus. J. Antimicrob. Chemother. 68, 4-11 (2013). 
30 McCurdy, S. P., Jones, R. N., Mendes, R. E., Puttagunta, S. \& Dunne, M. W. In vitro activity of dalbavancin against drug-resistant Staphylococcus aureus Isolates from a Global Surveillance Program. Antimicrob. Agents Chemother. 59, 5007-5009 (2015).

31 Boucher, H. W. et al. Once-weekly dalbavancin versus daily conventional therapy for skin infection. N. Engl. J. Med. 370, 2169-2179 (2014).

32 Two new drugs for skin and skin structure infections. Med. Lett. Drugs Ther. 56 73-75 (2014).

33 Raad, I. et al. Efficacy and safety of weekly dalbavancin therapy for catheter-related bloodstream infection caused by gram-positive pathogens. Clin. Infect. Dis. 40, 374-380 (2005).
34 Dunne, M. et al. Single Dose Treatment with Dalbavancin for Acute Bacterial Skin and Skin Structure Infection (ID Week, San Diego, CA, USA, 2015).

35 Dorr, M. B. et al. Human pharmacokinetics and rationale for once-weekly dosing of dalbavancin, a semi-synthetic glycopeptide. J. Antimicrob. Chemother. 55(Suppl 2), ii25-ii30 (2005).

36 Leighton, A. et al. Tolerability, pharmacokinetics, and serum bactericidal activity of intravenous dalbavancin in healthy volunteers. Antimicrob. Agents Chemother. 48 940-945 (2004).

37 Clinical and Laboratory Standards Institute (CLSI). Performance Standards for Antimicrobial Susceptibility Testing (CLSI, Wayne, PA, 2014). 